\title{
Acute Behavior of Oxygen Consumption, Lactate Concentrations, and Energy Expenditure During Resistance Training: Comparisons Among Three Intensities
}

\author{
Gustavo A. João ${ }^{1,2}$, Gustavo P. L. Almeida ${ }^{2 * t}$, Lucas D. Tavares ${ }^{1,3+}$, \\ Carlos Augusto Kalva-Filho ${ }^{4 t}$, Nelson Carvas Junior ${ }^{5 t}$, Francisco L. Pontes ${ }^{6 t}$, \\ Julien S. Baker ${ }^{7 \dagger}$, Danilo S. Bocalini ${ }^{8+}$ and Aylton J. Figueira ${ }^{2+}$
}

OPEN ACCESS

Edited by:

Patrik Drid,

University of Novi Sad, Serbia

Reviewed by:

Erika Zemková

Comenius University, Slovakia

Saša Krstulović,

University of Split, Croatia

*Correspondence:

Gustavo P. L. Almeida

ligegu@hotmail.com

tThese authors have contributed equally to this study

Specialty section:

This article was submitted to

Elite Sports and Performance

Enhancement,

a section of the journal

Frontiers in Sports and Active Living

Received: 19 October 2021 Accepted: 15 November 2021 Published: 15 December 2021

Citation:

João GA, Almeida GPL, Tavares $L D$,

Kalva-Filho CA, Carvas Junior N, Pontes FL, Baker JS, Bocalini DS and Figueira AJ (2021) Acute Behavior of

Oxygen Consumption, Lactate

Concentrations, and Energy

Expenditure During Resistance

Training: Comparisons Among Three

Intensities.

Front. Sports Act. Living 3:797604.

doi: 10.3389/fspor.2021.797604
' Department of Exercise Physiology Laboratory, Metropolitanas Unidas College, São Paulo, Brazil, ${ }^{2}$ Department of Translational Physiology Laboratory, São Judas Tadeu University, São Paulo, Brazil, ${ }^{3}$ Laboratory of Neuromuscular Adaptations to Strength Training, University of São Paulo, São Paulo, Brazil, ${ }^{4}$ Laboratory of Applied Sports Science, Institute of Physical Education and Sports, Federal University of Alagoas, Maceió, Alagoas, Brazil, ${ }^{5}$ Department of Evidence-Based Health, Brazilian Cochrane Center, University Federal de São Paulo, São Paulo, Brazil, ${ }^{6}$ Physical Activity and Aging Laboratory, School of Arts, Sciences and Humanities, University of São Paulo, São Paulo, Brazil, ${ }^{7}$ Centre for Health and Exercise Science Research, Hong Kong Baptist University, Kowloon Tong, Hong Kong, China, ${ }^{8}$ Experimental Physiology and Biochemistry Laboratory, Physical Education and Sport Center of Federal University of Espirito Santo, Vitoria, Brazil

Purpose: This study aimed to compare the oxygen consumption, lactate concentrations, and energy expenditure using three different intensities during the resistance training sessions.

Methods: A total of 15 men (22.9 \pm 2.61 years) experienced in resistance training underwent 3 sessions composed of 8 exercises (chest press, pec deck, squat, lat pull-down, biceps curl, triceps extension, hamstring curl, and crunch machine), which were applied in the same order. The weight lifted differed among the sessions [high session: 6 sets of 5 repetitions at 90\% of 1-repetition maximum (1-RM); intermediary session: 3 sets of 10 repetitions at 75\% of 1-RM; and low session: 2 sets of 15 repetitions at $60 \%$ of $1-\mathrm{RM}]$. The oxygen consumption $\left(\mathrm{VO}_{2}\right)$ - during and after (excess postexercise oxygen consumption (EPOC)) the session, blood lactate concentration, and energy expenditure (i.e., the sum of aerobic and anaerobic contributions, respectively) were assessed.

Results: The VO2 significantly decreased in the function of the weight lifting $\left(F_{(2.28)}\right.$ $\left.=17.02 ; p<0.01 ; \eta_{G}{ }^{2}=0.32\right)$. However, the aerobic contributions significantly increase in the function of the weight lifting $\left(F_{(2.28)}=79.18 ; p<0.01 ; \eta_{G}{ }^{2}=\right.$ 0.75). The anaerobic contributions were not different among the sessions $(p>$ 0.05; $\eta_{G}{ }^{2}<0.01$ ). Thus, the total energy expenditure during the session (kcal) significantly increased in the function of the weight lifting $\left(F_{(2.28)}=86.68 ; p<\right.$ $\left.0.01 ; \eta_{G}{ }^{2}=0.75\right)$. The energy expenditure expressed in time unit $\left(\mathrm{kcal} \cdot \mathrm{min}^{-1}\right)$ was higher in low session than in high session $\left(F_{(2.28)}=6.20 ; p<0.01 ; \eta_{G}{ }^{2}=0.15\right)$. 
Conclusion: The weight lifted during resistance training-induced different physiological responses, which induced higher energy expenditure per unit of time during the low session.

Keywords: EPOC, energy expenditure (EE), caloric cost, resistance training (RT), strength training

\section{INTRODUCTION}

Resistance training (RT) has been practiced by millions of people such as athletes and individuals interested in fitness, who seek increased health statuses, such as improving body composition, lipid profiles, and athletic performance via improvements in strength and muscle power (Nunez et al., 2019; GonzalezHernandez et al., 2020).

Data on the metabolic profiles of resistance training responses of the practitioner to oxygen consumption (VO2), aerobic and anaerobic contributions, blood lactate concentration, and energy expenditure (EE) remain unclear in the literature.

However, RT induces several adaptations and requires different metabolic demands (aerobic and anaerobic) during the session (Schoenfeld, 2013; Aguiar et al., 2018). However, understanding better how the variables such as the number of sets and repetitions and the intensities (weight lifted) could contribute to the metabolic system that can help to prescribe resistance training methodologies with accuracy.

In this context, demonstrating how different RT prescriptions influence metabolic stress (i.e., energy contributions) is important in practical and study approaches. Identification of RT sessions with higher EE-during and after exercise is useful for health purposes and body weight management (Da Silva et al., 2010; Kelleher et al., 2010; Nunez et al., 2019). Considering the size principle of motor unit recruitment, the lower threshold fibers (type I) are activated during submaximal contractions, while the higher threshold fibers (type II) are progressively recruited dependent on function and intensity (Garber et al., 2011; Morton et al., 2019). Thus, an increase in the EE is expected with increased increments in the weight lifted. Little study has investigated resistance training and metabolic systems and their energy cost. Thornton and Potteiger (2002) reported that RT sessions using 2 sets of 8 repetitions at $85 \%$ of maximal repetition [1-repetition maximum (1-RM)] produce greater EE-mainly after exercise-over 2 sets of 15 repetitions at $45 \%$ of 1 -RM.

Recently, Brunelli et al. (2019) investigated EE, energy system contributions after an acute low-load or high-load RT using two different loads: low-load RT (30\% of 1-RM) or a high-load RT ( $80 \%$ of $1-\mathrm{RM})$ during knee extension exercise. Exercise EE and the anaerobic lactic system contributions were significantly higher for $30 \%$ of $1-\mathrm{RM}$ compared to $80 \%$ of $1-\mathrm{RM}$.

Considering these results and the gap in the current literature, previous studies have not investigated intermediate intensities (for example, 75\% of 1-RM), and more exercises per session, as are often performed in practical RT sessions. Our hypothesis is that an RT session consisting of 8 exercises, associated with intensities $>80 \%$ of $1-\mathrm{RM}$, could promote an increase in EE.
TABLE 1 | Participants baseline characteristics and dietary intake (mean \pm SD).

\begin{tabular}{|c|c|}
\hline Age (years) & $22.87 \pm 2.61$ \\
\hline Time experience (years) & $3.27 \pm 1.53$ \\
\hline Body mass (kg) & $83.60 \pm 9.76$ \\
\hline Height (cm) & $183.07 \pm 5.60$ \\
\hline$\%$ Body Fat & $9.94 \pm 3.32$ \\
\hline Fat mass (kg) & $10.63 \pm 2.62$ \\
\hline Fat-free mass (kg) & $72.97 \pm 7.47$ \\
\hline BMl $\left(\mathrm{kg} / \mathrm{cm}^{2}\right)$ & $24.90 \pm 2.15$ \\
\hline RMR (kcal) & $2395.47 \pm 415.80$ \\
\hline Carbohydrate (g) & $482.00 \pm 46.63$ \\
\hline Protein (g) & $156.87 \pm 11.16$ \\
\hline Lipids (g) & $67.60 \pm 7.41$ \\
\hline Total (kcal) & $3325.73 \pm 245.15$ \\
\hline
\end{tabular}

Thus, the aim of this study was to compare EE using oxygen consumption values during and after RT sessions using intermediate (IS), high (HS), and low (LS) intensities, performed using 30 repetitions.

\section{MATERIALS AND METHODS}

\section{Participants}

A total of 15 healthy young men with at least 12 months of experience in RT were recruited and assigned to a randomized trial (Table 1). All the procedures were approved by the Ethical Institutional Review Board of São Judas Tadeu University (protocol: 2.022.898) and conducted according to the principles expressed in the Declaration of Helsinki. The volunteers were told to refrain from any resistance exercise during the period of the experiment; all the participants read and signed an informed consent document.

The following criteria were established as exclusion: (1) smokers; (2) submitted to diets designed for both the reduction of body mass and/or muscle mass increase; (3) free of any metabolic disorders with no medication intake that affects EE (sympathomimetics, bronchodilators, antidepressants, amphetamines, and illicit drugs); (4) muscle or tendon injury in the last 3 months; (5) being under the treatment of infectious disease; (6) having used in the last 6 months or be using any type of ergogenic agent of hormonal origin to increase strength or hypertrophy; and (7) no change in diet as reported by the food recall questionnaire. Inclusion criteria were as follows: (1) minimum experience of 12 months in RT; (2) reports of minimum training frequencies of 3 times per week; (3) present resting systolic blood pressure between 120 and $129 \mathrm{~mm}$ $\mathrm{Hg}$ and diastolic blood pressure between 80 and $84 \mathrm{~mm} \mathrm{Hg}$ 


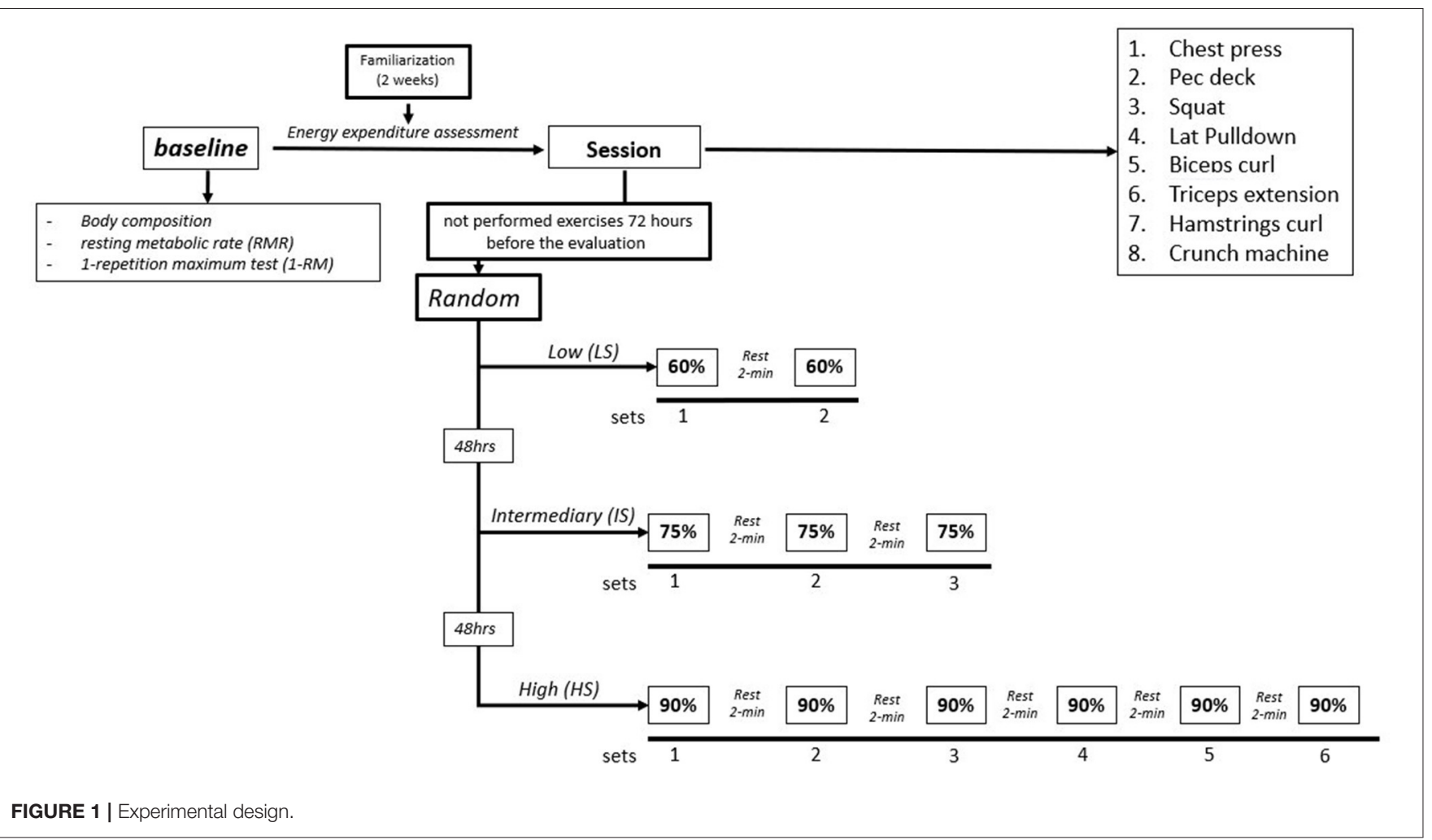

(Pescatello et al., 2004), assessed immediately before the experimental sessions through an auscultatory method; and (4) demonstration of a medical declaration that guarantees healthy clinical status to participate. All the individuals participating in this study answered the sociodemographic questionnaire with open questions about physical activity history, nutritional information, disease history, and family aspects.

\section{Experimental Design}

The experimental design was composed of 6 visits. In the first visit, body composition and 1 RM were assessed. A familiarization with each exercise using the equipment and measurements (e.g., the mask of the gas analyzer) was performed during the second visit. In the third visit, the resting metabolic rate (RMR) was assessed for 30-min in a supine position. After 2 weeks, during the 3 lasted visits, participants underwent the experimental RT sessions composed of 8 exercises, which were performed at low ( $60 \%$ of 1-RM; LS), intermediary (75\% of 1RM; IS), and high (90\% of 1-RM; HS) intensities. The choice of intensities used reflects most RT methods and was based on the American College of Sports Medicine (ACSM) and the National Strength and Conditioning Association (NSCA) recommendations (Fry, 2004; Fragala et al., 2019). The sessions were applied in random order and separated by $48 \mathrm{~h}$ of recovery (Figure 1).

In the familiarization session, the weight lifted was adjusted to perform 1 set of 20 repetitions. All the participants were informed about how to correctly perform the exercises and were under the direct supervision of the study team. The $\mathrm{VO}_{2}$ was monitored constantly and the blood lactate concentrations were obtained at rest and after experimental sessions (i.e., immediately and after 5, 10,15 , and 20 min of recovery).

The RT was strictly controlled so that individuals only exercised under the direct supervision of an experienced strength and conditioning professional. Individuals were not allowed to perform any other RT exercises during this period. All the participants were instructed not to consume coffee $12 \mathrm{~h}$ before the evaluations. In addition, all the participants did not exercise for at least $48 \mathrm{~h}$ prior to evaluation according to a study by Brigatto et al. (2019). All the subjects were monitored continuously using telephone calls and personal interviews to ensure adherence. No participants reported signals of muscle microtrauma (e.g., muscle pain) or conducting strenuous sessions $72 \mathrm{~h}$ before the experiments. Nutrient intake was assessed using a 24 -h food recall on 2 non-consecutive weekdays and 1 weekend day.

\section{Body Composition}

Body mass (BM) was measured using the G-Tech ${ }^{\circledR}$ (Hospital Medical Products - ACCUMED / serial: 47110100965, China) scale with an accuracy of $0.100 \mathrm{~g}$ with the individuals positioned barefoot and with minimum possible clothing. Height $(\mathrm{H})$ was measured using a Sanny ${ }^{\circledR}$ stadiometer with a precision of $0.1 \mathrm{~cm}$. BM index (BMI) was obtained using the same equation: $\mathrm{BM} /\left(\mathrm{H}^{2}\right)$ and circumferences were measured using a standard protocol outlined previously (Cavedon et al., 2018). To assess body composition and subcutaneous fat thickness, an ultrasound imaging unit was used (BodyMetrix ${ }^{\circledR}$ PRO System, Intelametrix, 
TABLE 2 | Test of 1 maximum repetition and load used in each exercise (mean \pm SD).

\begin{tabular}{|c|c|c|c|c|}
\hline Exercises & $\begin{array}{c}\text { Test } \\
\text { 1-RM (kg) }\end{array}$ & $\begin{array}{c}\text { LS } \\
60 \% \text { of } 1-\mathrm{RM}(\mathrm{kg})\end{array}$ & $\begin{array}{c}\text { IS } \\
75 \% \text { of } 1-\mathrm{RM}(\mathrm{kg})\end{array}$ & $\begin{array}{c}\text { HS } \\
90 \% \text { of 1-RM (kg) }\end{array}$ \\
\hline Chest press & $93 \pm 23$ & $56 \pm 14$ & $70 \pm 17$ & $84 \pm 21$ \\
\hline Pec deck & $80 \pm 19$ & $48 \pm 11$ & $60 \pm 14$ & $72 \pm 17$ \\
\hline Squat & $103 \pm 27$ & $62 \pm 16$ & $78 \pm 20$ & $93 \pm 24$ \\
\hline Lat pull-down & $105 \pm 19$ & $63 \pm 11$ & $79 \pm 14$ & $94 \pm 17$ \\
\hline Biceps curl & $47 \pm 9$ & $28 \pm 60$ & $35 \pm 70$ & $42 \pm 90$ \\
\hline Triceps extension & $93 \pm 13$ & $56 \pm 80$ & $70 \pm 10$ & $84 \pm 12$ \\
\hline Hamstrings curl & $111 \pm 18$ & $66 \pm 11$ & $83 \pm 14$ & $100 \pm 17$ \\
\hline Crunch machine & $108 \pm 14$ & $65 \pm 90$ & $81 \pm 11$ & $97 \pm 13$ \\
\hline
\end{tabular}

Low (60\% of 1-RM; LS), intermediary (75\% of 1-RM; IS), and high-intensities (90\% of 1-RM; HS).

Livermore, California, USA-BodyView ${ }^{\mathrm{TM}}$ software) with a wave frequency of $2.5 \mathrm{MHz}$ (Selkow et al., 2011). The ultrasound probe was applied perpendicularly to the skin for measurement. A water-soluble gel was used on the transducer to help acoustic coupling and avoid excessive skin pressure. The individuals were instructed to fast for $3 \mathrm{~h}$ before testing. The pretest and posttest evaluations were performed at the same time. Imaging was performed on the right side of the bodies of individuals and to further ensure the accuracy of the assessments, at least 3 pictures were taken. The average of the 3 assessments was used for statistical analysis.

\section{1-Repetition Maximum Test}

Maximum dynamic strength was assessed using a 1-RM test for the following exercises: chest press, pec deck, squat, lat pull-down, biceps curl, triceps extension, hamstring curl, and crunch machine (fitness line equipment, GervaSport ${ }^{\circledR}$, Spain), all accomplished in the pieces of equipment. The testing protocol followed previous recommendations by Haff and Triplett (Haff and Triplett, 2015). Participants reported to the laboratory having refrained from any exercise other than activities of daily living for at least $72 \mathrm{~h}$ before testing.

In brief, participants warmed up for $5 \mathrm{~min}$ on a treadmill (Movement Technology, São Paulo, Brazil) at $60 \%$ of maximum heart rate. During the first set, participants performed 5 repetitions at $\sim 50 \%$ of the estimated 1 -RM followed by 1 set of 3 repetitions at $\sim 60-80 \%$ of the estimated $1-\mathrm{RM}$ with a 3-min recovery interval between sets. After the warmup sets, participants had 5 attempts to find their 1-RM with 3-min intervals between trials. The 1-RM was deemed as the maximum weight that could be lifted no more than once using the correct technique. Verbal encouragement was given throughout the testing. All the testing sessions were supervised by the study team and deemed valid (Table 2).

The test-retest interclass correlation coefficient (ICC) and standard error of measurement (SEm) from our laboratory for 1-RM were applied to all the exercises. Therefore, test-retest

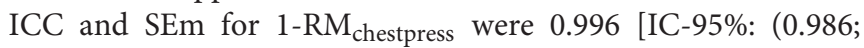
0.999)] with SEm (0.125) and test-retest for $1-\mathrm{RM}_{\text {squat }}$ was 0.998 [IC-95\%: (0.986; 0.999)] with SEm (0.031) (Table 2).

\section{Experimental Sessions}

The participants performed a warm-up for $5 \mathrm{~min}$ before all the protocols on a treadmill (Movement Technology, São Paulo, Brazil) at $60 \%$ of maximum heart rate. After that, the participants performed 20 repetitions at $50 \%$ of $1-\mathrm{RM}$ for each one of the exercises. After the warmup sets, the participants rested for $5 \mathrm{~min}$ and then started the protocols. All the sessions were composed of 8 exercises (chest press, pec deck, squat, lat pull-down, biceps curl, triceps extension, hamstrings curl, and crunch machine) performed sequentially. The participants in the sessions LS (60\% of $1-\mathrm{RM})$ were encouraged to reach 15 repetitions in 2 sets, IS (75\% of $1-\mathrm{RM})$ were encouraged to reach 10 repetitions in the 3 sets; and HS (90\% of 1-RM) were encouraged to reach 5 repetitions in the 6 sets. The interest and interexercise rest was $120 \mathrm{~s}$ during all the protocols. The load was defined based on previous tests of a 1-RM. Thus, the load was not changed throughout the session.

In all the protocols, the cadence of repetitions was carried out in a controlled fashion, with phase concentric and eccentric muscle actions of $\sim 1.5 \mathrm{~s}$, for a total repetition duration of $\sim 3 \mathrm{~s}$ (controlled by a metronome).

\section{Internal Responses}

The $\mathrm{VO}_{2}$ during the $\mathrm{RT}$ sessions was measured using a gas analyzer (Fitmate Pro; $\operatorname{COSMED}^{\circledR}$, Rome, Italy) with a flexible flowline as described previously (Lee et al., 2011). The gas analyzer was calibrated following the specifications of the manufacturer before each test. Immediately after completion of the exercise protocol, EPOC was measured for a total of $30 \mathrm{~min}$ in a supine position. The $\mathrm{VO}_{2}$ of the participants was obtained every $2 \mathrm{~min}$. The area under the curve was assumed as the aerobic energy (AE) equivalent during the different experimental sessions. The integral was obtained through the software Origin (OriginPro 8.0, OriginLab Corporation, Microcal, Massachusetts, USA).

The lactate concentration was determined using a lactimeter model Accusport Plus Roche ${ }^{\circledR}$, following the recommendation from previous studies (Foxdal et al., 1990; Franchini et al., 2004). Blood samples were taken from a finger capillary before the protocol (i.e., at rest); monitored immediately; and 5, 10, 15, and $20 \mathrm{~min}$ after RT session. To obtain anaerobic energy (ANE) 
TABLE 3 | External responses during different experimental sessions prescribed at low (60\% of 1-RM; LS), intermediary (75\% of 1-RM; IS), and high-intensities (90\% of 1-RM; HS) (mean $\pm \mathrm{SD}$ ).

\begin{tabular}{|c|c|c|c|}
\hline Parameters & LS & IS & HS \\
\hline Absolute-load (kg) & $9858 \pm 4526[7352 ; 12365]{ }^{\star} t$ & $15028 \pm 2274$ [13769; 16287] & $17570 \pm 4764$ [14932; 20208] \\
\hline Duration the session (min) & $44.00 \pm 3.02[42 ; 46]{ }^{\star} t$ & $61.40 \pm 3.27[59 ; 63]^{*}$ & $116.00 \pm 4.09[113 ; 118]$ \\
\hline
\end{tabular}

Mean $\pm S D$, standard deviation; $C l$, confidence interval.

Absolute-load $=$ no: of sets $x$ no. of repetitions $\times$ weight lifted $(\mathrm{kg})$.

${ }^{*} p<0.05$ vs. HS $(90 \%$ of $1-R M)$.

$t_{p}<0.05$ vs. IS (75\% of $\left.1-R M\right)$.

equivalent, data were analyzed as the difference between peak and baseline values $[\mathrm{La} \Delta]$, using the equivalent of $3 \mathrm{ml} \cdot \mathrm{kg}^{-1}$ of oxygen for each unit of lactate accumulated (Hunter and Byrne, 2005; Bertuzzi et al., 2016). The AE, ANE, and EPOC data were converted into calorie units using the equivalent of 5.05 calories (kcal) per liter of oxygen consumed (Phillips and Ziuraitis, 2003). Thus, the total energy equivalents were assumed as the sum of $\mathrm{AE}, \mathrm{ANE}$, and EPOC.

\section{Load Control}

The "absolute load" for each exercise performed was calculated using the total weight lifted during the training period (i.e., no. of sets $\times$ no. of repetitions $\times$ weight lifted per repetitions) (Scott and Reis, 2016).

\section{Statistical Analyses}

The sample size required was estimated using $\mathrm{G}^{*}$ Power 3.1 software (version 3.1.9.4, Heinrich-Heine-University, Germany) (Faul et al., 2007), using effect sizes from a previous study comparing EE of different intensities during set RT (Scott et al., 2010). A priori power analysis assuming an estimating error of $\alpha$ $=0.05$ and power $=80 \%$ to an actual power was 0.86 suggested a sample size of 8 participants to achieve a statistically significant difference between intensities. Thus, the use of $n=15$ enabled a statistical power $=1.80$.

However, 15 individuals were initially assigned to perform RT sessions with different intensities. Repeated measures ANOVA were used to analyze EE values, followed by Bonferroni's post-hoc test, where necessary. Normality, homogeneity, and sphericity assumptions were confirmed with Shapiro-Wilk, Levene's, and Mauchly's tests, respectively. Case of spherical (homogeneous) nonprincipal values used Greenhouse-Geisser correction. The generalized eta square $\left(\eta_{\mathrm{G}}{ }^{2}\right)$ was used as effect size and interpreted according to Cohen. For the CI for intraclass correlation, calculations using a (psychometric) package in the statistic program R software. Therefore, CI was then calculated at the desired level using formulas provided by McGraw and Wong (1996) and Bliese (2000). Overall data are presented as means, SDs, and 95\% CI. Alpha was set at 0.05 . The data were processed in $\mathrm{R}$ version 1.0.44 for Macintosh software.

\section{RESULTS}

The test-retest ICC and SEm from our laboratory for 1-RM were applied to the main exercises. Therefore, test-retest ICC and SEm for $1-\mathrm{RM}_{\text {chestpress }}$ were 0.996 [IC-95\%: $\left.(0.986 ; 0.999)\right]$ with SEm (0.125) and test-retest for $1-\mathrm{RM}_{\text {squat }}$ was 0.998 [IC-95\%: (0.986; 0.999)] with SEm (0.031).

Table 3 demonstrates the external responses of the different intensities in the RT. The absolute load was lower in the LS intensity when compared to IS and HS $\left(F_{(2.28)}=16.77 ; p<\right.$ $\left.0.01 ; \eta_{\mathrm{G}}{ }^{2}=0.40\right)$. However, there were no significant differences between IS and HS. Due to absolute load, there were observed significant differences among duration of the sessions $\left(F_{(2.28)}=\right.$ $\left.2,773.34 ; p<0.01 ; \eta_{\mathrm{G}}^{2}=0.98\right)$.

Table 4 demonstrates the internal responses during the different intensities in RT. The $\mathrm{VO}_{2}$ was higher during LS when compared with other intensities $\left(F_{(2.28)}=17.02 ; p<0.01 ; \eta_{\mathrm{G}}{ }^{2}\right.$ $=0.32$ ). There were no significant differences in $\mathrm{VO}_{2}$ during the different intensities of HS when compared to IS. There were no significant differences in lactate concentrations between intensities of RT $\left(F_{(2.28)}=0.25 ; p=0.77 ; \eta_{\mathrm{G}}{ }^{2}=0.01\right)$. There was a significant difference in AE in the HS when compared with LS and IS $\left(F_{(2.28)}=79.18 ; p<0.01 ; \eta_{\mathrm{G}}{ }^{2}=0.75\right)$. There were no significant differences in the ANE (L) among intensities during sessions $\left(F_{(2.28)}=0.38 ; p=0.68 ; \eta_{\mathrm{G}}{ }^{2}=0.01\right)$. The EPOC values were significantly lower in IS when compared LS and HS $\left(F_{(2.28)}\right.$ $=5.57 ; p<0.01 ; \eta_{\mathrm{G}}{ }^{2}=0.12$ ).

The EE of intensities is given in Figure 2. A significant difference in total $\mathrm{EE} \mathrm{(kcal)} \mathrm{was} \mathrm{found} \mathrm{between} \mathrm{all} \mathrm{the}$ intensities $\left(F_{(2.28)}=86.68 ; p<0.01 ; \eta_{\mathrm{G}}{ }^{2}=0.75\right)$. Additionally, significant differences were found in EE expressed per time unit $\left(\mathrm{kcal} \cdot \mathrm{min}^{-1}\right)$ only between LS $(6.49 \pm 1.01)$ and HS $(5.27 \pm 1.03)$ $\left(F_{(2.28)}=6.20 ; p<0.01 ; \eta_{\mathrm{G}}^{2}=0.16\right)$.

\section{DISCUSSION}

The purpose of this study was to compare the oxygen consumption, lactate concentrations, and EE using three different intensities (LS, IS, and HS) during the RT sessions. The main results demonstrated that HS presented higher values of EE, which was accompanied by a greater duration of the session and higher values of AE. However, when EE during sessions was expressed per time unit $\left(\mathrm{kcal} \cdot \mathrm{min}^{-1}\right)$ of the session, higher values emerged during LS when compared with the HS. In addition, although the weight lifted had been different, the ANE was similar among the sessions.

According to the previous studies (Hunter et al., 2003; Haddock and Wilkin, 2006; Reis et al., 2017; Martorelli et al., 2021), the total EE increases when higher weights were lifted 
TABLE 4 | The outcome of the lactate, energy expenditure and EPOC during different experimental sessions prescribed at low (60\% of 1-RM; LS), intermediary (75\% of $1-\mathrm{RM} ; \mathrm{IS})$ and high-intensities (90\% of 1-RM; HS) (mean $\pm \mathrm{SD})$.

\begin{tabular}{|c|c|c|c|}
\hline Parameters & LS & IS & HS \\
\hline \multicolumn{4}{|l|}{ Internal responses } \\
\hline $\mathrm{VO}_{2}\left(L \cdot \mathrm{min}^{-1}\right)$ & $1.61 \pm 0.26[1.47 ; 1.75]^{\star} t$ & $1.38 \pm 0.32[1.20 ; 1.56]$ & $1.15 \pm 0.25[1.01 ; 1.29]$ \\
\hline \multicolumn{4}{|c|}{ Energy equivalents } \\
\hline $\mathrm{AE}(\mathrm{L})$ & $42.80 \pm 6.70[39.09 ; 46.51]^{\star} t$ & $59.25 \pm 15.12[50.88 ; 67.62]$ * & $107.12 \pm 22.83[94.48 ; 119.76]$ \\
\hline Total (L) & $56.16 \pm 7.32[52.11 ; 60.21]^{*} t$ & $71.25 \pm 15.48[62.68 ; 79.82]^{*}$ & $120.84 \pm 22.83[108.20 ; 133.48]$ \\
\hline
\end{tabular}

Mean $\pm S D$, standard deviation; $C l$, confidence interval; La $\triangle$, difference between lactate peak and baseline; AE, aerobic energy equivalent; ANE, anaerobic energy equivalent; EPOC, excess post-exercise oxygen consumption. Total $=A E(L)+A N E(L)+E P O C(L)$.

${ }^{*} p<0.05$ vs. HS $(90 \%$ of $1-R M)$.

$t_{p}<0.05$ vs. IS $(75 \%$ of $1-R M)$.
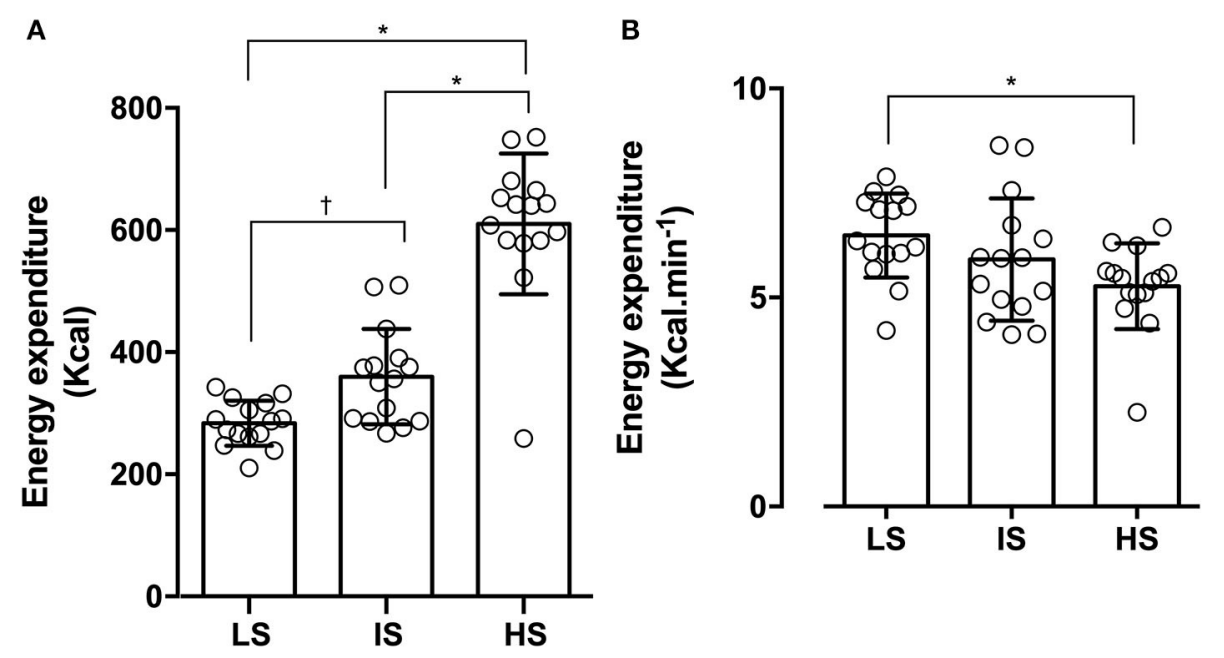

FIGURE 2 | (A) Energy expenditure and (B) energy expenditure during different experimental sessions prescribed at low (60\% of 1-RM; LS), intermediary (75\% of 1-RM; IS) and high-intensities (90\% of $1-\mathrm{RM}$; HS). ${ }^{*} p<0.05$ vs. HS (90\% of $\left.1-\mathrm{RM}\right){ }^{\dagger} p<0.05$ vs. IS (75\% of $\left.1-\mathrm{RM}\right)$.

during the sessions. Hunter et al. (2003) compared EE using low $(25 \%$ of $1-\mathrm{RM})$ or moderate intensities (65\% of $1-\mathrm{RM})$ prescribed with the same total time $(29 \mathrm{~min})$ demonstrating that energy expenditure was $45 \%$ higher during the moderateintensity session. Some of the studies use fixed repetitions during the sets, which limited direct comparisons of our results, mainly because the length of sessions acts as an inherent variable of the $\mathrm{EE}$ assessments.

Brunelli et al. (2019) demonstrated higher EE during the exercise performed at low-intensity (30\% of 1-RM) compared with high-intensity ( $85 \%$ 1-RM) sessions. These results were accompanied by higher contributions from the anaerobic lactic pathway, indicating that the longest time in exercise compensated for the differences in the weight lifted during the sessions. Although the abovementioned article used only the knee extension machine, our results used 8 exercises and also demonstrated the higher values of EE per min during the LS compared with the HS. However, we could not demonstrate significant differences in ANE values, indicating that the higher values of $\mathrm{EE}$ in the LS were associated with the length of the session-that directly influenced the area of $\mathrm{VO}_{2}$ and not with changes in metabolic balance. A possible explanation of these differences between our results and those observed by Brunelli et al. (2019) may be the physical conditioning level of the participants. A study by Brunelli et al. (2019) was conducted with men with an inactive lifestyle who had not participated in regular resistance exercise programs in the past 12 months. On the other hand, in this study, one of the inclusion criteria of the participants was to have a minimum experience of 12 months in the RT. However, direct comparisons between these studies are limited because we did not use knee extension during our sessions and our RT sessions consisted of 8 exercises, while a study by Brunelli et al. (2019) only used 1 exercise modality.

The magnitude of the EPOC was measured for $30 \mathrm{~min}$ after the session, mainly considering that the participants returned to baseline approximately within $25 \mathrm{~min}$ (Farinatti 
et al., 2009; Ratamess et al., 2014). The EPOC may be the result of energy-requiring processes such as the replenishment of myoglobin oxygen stores, adenosine triphosphate, creatine phosphate, increased ventilation, heart rate, body temperature, triglyceride or fatty acid cycling, substrate utilization shifts from carbohydrates to fats, and mitochondrial activity (Borsheim and Bahr, 2003; Abboud et al., 2013). Our results indicated that the weight lifted between sessions can influence differently in these processes and corroborated previous studies using RT (Haddock and Wilkin, 2006; Farinatti et al., 2009; Abboud et al., 2013; Ratamess et al., 2014). On the other hand, in addition to the weight lifted, the session duration could cause the biggest physiological disorders induced by HS, related to the EPOC values. A study by Abboud et al. (2013) showed that during RT with a high absolute load $(20,000 \mathrm{~kg})$, the participants expended significantly more energy $(484 \pm 29 \mathrm{kcal})$ than the low absolute load $(10,000 \mathrm{~kg})(247 \pm 18 \mathrm{kcal})$ and supported this study.

The methods to estimate the EEs may also contribute to value variation. In this context, this study used the area under the curve of the $\mathrm{VO}_{2}$ and lactate accumulation to estimate the $\mathrm{AE}$ and ANE, respectively.

Although these procedures were previously used in the context of RT, Brunelli et al. (2019) and Zagatto et al. (2016) demonstrated their validity and applicability and some limitations should be noticed in this study. First, it is preferable to use breath-by-breath assessments of $\mathrm{VO}_{2}$ to estimate the $\mathrm{AE}$, allowing measurements with high precision during effort and recovery (here the $\mathrm{VO}_{2}$ was assessed through means every $2 \mathrm{~min}$.). Second, this device limitation also precludes the determination of the anaerobic lactic equivalents through the fast phase of the EPOC, underestimating the total anaerobic equivalents. Third, although no differences were observed for ANE, the determination of lactate concentrations only after the session may underestimate the EEs, mainly because of lactate removal during recovery intervals. Therefore, collections after each exercise would be more accurate, as lactate would not be removed during recovery and, thus, the contribution would be greater. Four, an intense workout of $48 \mathrm{~h}$ before the evaluation could influence the performance of the participants because of muscle microtrauma. A low intensity workout of $48 \mathrm{~h}$ before will not influence the performance of the participants. We monitor the exercise routines $48 \mathrm{~h}$ before our experiments, as previously used for the same purpose (Brigatto et al., 2019). However, no participants reported signs of muscle microtrauma (e.g., muscle pain) or strenuous sessions $72 \mathrm{~h}$ before the experiments.

Together these methodological limitations possibly underestimated the AE, ANE, and EPOC values of the different RT sessions, it directly limits comparisons with previous studies. Even considering these limitations and the differences between studies, the results of this study seemed to be within the range of EE previously reported. In addition, this study used the absolute load method to determine the training load, which does not consider all the training variables (e.g., repetition velocity). We opted to use the absolute load method, mainly considering its practical application, outlined in a previous study (Scott and Reis, 2016). Future studies should advance the knowledge related to energy systems contribution using more robust approaches to equalize the absolute loads of the sessions.

\section{CONCLUSION}

The results of this study advance the knowledge related to EE during the RT, demonstrating that when the EE during sessions was expressed per time unit $\left(\mathrm{kcal} \cdot \mathrm{min}^{-1}\right)$ of the session, the LS presented higher values than HS. Thus, our results indicated that the duration of the exercise sessiontherefore the session length-induced greater influence on EE values than different energy balances (e.g., higher anaerobic contributions), at least when the sets were performed without fixed repetitions.

\section{PRACTICAL APPLICATION}

In the context of applied practice, this information can be valuable for athletes, exercise physiologists, enthusiasts, and fitness professionals who know and understand with accuracy the contribution of the metabolic system during the RT sessions consisting of 8 exercises and different intensities (low, intermediate, and high). As a result, individuals can select the best option to achieve specific outcomes, for example, losing weight, gaining muscle mass, or a combination of both options.

\section{DATA AVAILABILITY STATEMENT}

The original contributions presented in the study are included in the article/supplementary material, further inquiries can be directed to the corresponding author/s.

\section{ETHICS STATEMENT}

The studies involving human participants were reviewed and approved by Ethical Institutional Review Board of São Judas Tadeu University (protocol: 2.022.898). The patients/participants provided their written informed consent to participate in this study.

\section{AUTHOR CONTRIBUTIONS}

GJ and NC performed the work's statistics. JB, AF, DB and CK-F developed the manuscript. GA and FP conducted article researches and data collection. GA and GJ structured the study design. All authors of the research had an important contribution to carry out the study. 


\section{REFERENCES}

Abboud, G. J., Greer, B. K., Campbell, S. C., and Panton, L. B. (2013). Effects of load-volume on EPOC after acute bouts of resistance training in resistance-trained men. J. Strength Cond. Res. 27, 1936-1941. doi: 10.1519/JSC.0b013e3182772eed

Aguiar, S. S., Sousa, C. V., Simoes, H. G., Neves, R. V. P., Costa, F., and de Souza, M. K. (2018). Acute metabolic responses following different resistance exercise protocols. Appl. Physiol. Nutr. Metab. 43, 838-843. doi: 10.1139/apnm-2017-0771

Bertuzzi, R., Melegati, J., Bueno, S., Ghiarone, T., Pasqua, L. A., and Gaspari, A. F. (2016). GEDAE-LaB: A free software to calculate the energy system contributions during exercise. PLoS ONE. 11:e0145733. doi: 10.1371/journal.pone. 0145733

Bliese, P. (2000). "Within-group agreement, non-independence, and reliability: Implications for data aggregation and analysis," in Multilevel Theory, Research and Methods in Organizations: Foundations, Extensions, and New Directions, eds K. J. Klein and S. W. J. Kozlowski (San Francisco: Jossey-Bass), 349-381.

Borsheim, E., and Bahr, R. (2003). Effect of exercise intensity, duration and mode on post-exercise oxygen consumption. Sports Med. 33, 1037-1060. doi: 10.2165/00007256-200333140-00002

Brigatto, F. A., Lima, L. E. M., Germano, M. D., Aoki, M. S., Braz, T. V., and Lopes, C. R. (2019). High resistance-training volume enhances muscle thickness in resistance-trained men. J. Strength Cond. Res. 15:313. doi: $10.1519 /$ JSC. 0000000000003413

Brunelli, D. T., Finardi, E. A. R., Bonfante, I. L. P., Gaspari, A. F., Sardeli, A. V., and Souza, T. M. F. (2019). Acute low- compared to high-load resistance training to failure results in greater energy expenditure during exercise in healthy young men. PLoS ONE. 14:e0224801. doi: 10.1371/journal.pone.0224801

Cavedon, V., Zancanaro, C., and Milanese, C. (2018). Anthropometric prediction of DXA-measured body composition in female team handball players. PeerJ. 6:e5913. doi: 10.7717/peerj.5913

Da Silva, R., Brentano, M., and Kruel, L. (2010). Effects of different strength training methods on postexercise energetic expenditure. J. Strength Cond. Res. 24:8. doi: 10.1519/JSC.0b013e3181aff2ba

Farinatti, P. T., Simao, R., Monteiro, W. D., and Fleck, S. J. (2009). Influence of exercise order on oxygen uptake during strength training in young women. J. Strength Cond. Res. 23, 1037-1044. doi: 10.1519/JSC.0b013e3181a2b3e4

Faul, F., Erdfelder, E., Lang, A.-G., and Buchner, A. (2007). G*Power 3: A flexible statistical power analysis program for the social, behavioral, and biomedical sciences. Behav. Res. Methods. 39, 175-191. doi: 10.3758/BF03193146

Foxdal, P., Sjodin, B., Rudstam, H., Ostman, C., Ostman, B., and Hedenstierna, G. C. (1990). Lactate concentration differences in plasma, whole blood, capillary finger blood and erythrocytes during submaximal graded exercise in humans. Eur. J. Appl. Physiol. Occup. Physiol. 61, 218-222. doi: 10.1007/BF00357603

Fragala, M. S., Cadore, E. L., Dorgo, S., Izquierdo, M., Kraemer, W. J., and Peterson, M. D. (2019). Resistance training for older adults: position statement from the national strength and conditioning association. J. Strength Cond. Res. 33, 2019-2052. doi: 10.1519/JSC.0000000000003230

Franchini, E., Matsushigue, K. A., and Colantonio, E., Kiss MAPDm. (2004). Comparison of accusport and yellow springs lactate analyzers. $R$. bras. Ci. e Mov. 12, 39-44. doi: 10.18511/RBCM.V12I1.540

Fry, A. C. (2004). The role of resistance exercise intensity on muscle fibre adaptations. Sports Med. 34, 663-679. doi: 10.2165/00007256-200434100-00004

Garber, C. E., Blissmer, B., Deschenes, M. R., Franklin, B. A., Lamonte, M. J., and Lee, I. M. (2011). American College of Sports Medicine position stand. Quantity and quality of exercise for developing and maintaining cardiorespiratory, musculoskeletal, and neuromotor fitness in apparently healthy adults: guidance for prescribing exercise. Med. Sci. Sports Exerc. 43, 1334-1359. doi: 10.1249/MSS.0b013e318213fefb

Gonzalez-Hernandez, J. M., Garcia-Ramos, A., Castano-Zambudio, A., CapeloRamirez, F., Marquez, G., and Boullosa, D. (2020). Mechanical, metabolic, and perceptual acute responses to different set configurations in full squat. J. Strength Cond. Res. 34, 1581-1590. doi: 10.1519/JSC.00000000000 02117

Haddock, B. L., and Wilkin, L. D. (2006). Resistance training volume and post exercise energy expenditure. Int. J. Sports Med. 27, 143-148. doi: $10.1055 / \mathrm{s}-2005-865601$
Haff, G., and Triplett, N. (2015). Principles of test selection and administration," in Essentials of Strength Training and Conditioning. Champaign IHK, editor.: Champaign, IL: Human Kinetics.

Hunter, G., and Byrne, N. (2005). Physical activity and muscle function but not resting energy expenditure impact on weight gain. $J$. Strength Cond. Res. 19, 225-230. doi: 10.1519/00124278-20050200000037

Hunter, G. R., Seelhorst, D., and Snyder, S. (2003). Comparison of metabolic and heart rate responses to super slow vs. traditional resistance training. J. Strength Cond. Res. 17, 76-81. doi: 10.1519/00124278-20030200000013

Kelleher, A. R., Hackney, K. J., Fairchild, T. J., Keslacy, S., and Ploutz-Snyder, L. L. (2010). The metabolic costs of reciprocal supersets vs. traditional resistance exercise in young recreationally active adults. J. Strength Cond. Res. 24, 1043-1051. doi: 10.1519/JSC.0b013e3181d3e993

Lee, J. M., Bassett, D. R. Jr., Thompson, D. L., and Fitzhugh, E. C. (2011). Validation of the Cosmed Fitmate for prediction of maximal oxygen consumption. J. Strength Cond. Res. 25, 2573-2579. doi: 10.1519/JSC.0b013e31 $81 \mathrm{fc} 5 \mathrm{c} 48$

Martorelli, A. S., De Lima, F. D., Vieira, A., Tufano, J. J., Ernesto, C., and Boullosa, D. (2021). The interplay between internal and external load parameters during different strength training sessions in resistance-trained men. Eur. J. Sport Sci. 21, 16-25. doi: 10.1080/17461391.2020.1725646

McGraw, K. O., and Wong, S. P. (1996). Forming some inferences about some intraclass correlation coefficients. Psychol. Methods 1, 30-46. doi: 10.1037/1082-989X.1.1.30

Morton, R. W., Sonne, M. W., Farias Zuniga, A., Mohammad, I. Y. Z., Jones, A., and McGlory, C. (2019). Muscle fibre activation is unaffected by load and repetition duration when resistance exercise is performed to task failure. J. Physiol. 597, 4601-4613. doi: 10.1113/ JP278056

Nunez, T. P., Amorim, F. T., Beltz, N. M., Mermier, C. M., Moriarty, T. A., and Nava, R. C. (2019). Metabolic effects of two high-intensity circuit training protocols: does sequence matter? J. Exerc. Sci. Fit. 18, 14-20. doi: 10.1016/j.jesf.2019.08.001

Pescatello, L. S., Franklin, B. A., Fagard, R., Farquhar, W. B., Kelley, G. A., and Ray, C. A. (2004). American College of Sports Medicine position stand. exercise and hypertension. Med. Sci. Sports Exerc. 36, 533-553. doi: 10.1249/01.MSS.0000115224.88514.3A

Phillips, W., and Ziuraitis, J. R. (2003). Energy cost of the ACSM singleset resistance training protocol. J. Strength Cond. Res. 17, 350-355. doi: 10.1519/00124278-200305000-00023

Ratamess, N., Rosenberg, J., Kang, J., Sundberg, S., Izer, K., and Levowsky, J. (2014). Acute oxygen uptake and resistance exercise performance using different rest interval lengths: The influence of maximal aerobic capacity and exercise sequence. J. Strength Cond. Res. 28, 1875-1888. doi: 10.1519/JSC.0000000000000485

Reis, V. M., Garrido, N. D., Vianna, J., Sousa, A. C., Alves, J. V., and Marques, M. C. (2017). Energy cost of isolated resistance exercises across low- to high-intensities. PLOS ONE. 12:e0181311. doi: 10.1371/journal.pone.01 81311

Schoenfeld, B. J. (2013). Potential mechanisms for a role of metabolic stress in hypertrophic adaptations to resistance training. Sports Med. 43, 179-194. doi: 10.1007/s40279-013-0017-1

Scott, B. C., and Reis, V. M. (2016). Training monitoring for resistance exercise: theory and applications. Sports Med. 46, 687-698. doi: 10.1007/s40279-015-0454-0

Scott, C. B., Leighton, B. H., Ahearn, K. J., and McManus, J. J. (2010). Aerobic, anaerobic, and excess postexercise oxygen consumption energy expenditure of muscular endurance and strength: 1-set of bench press to muscular fatigue. J. Strength Cond. Res. 25, 903-908. doi: 10.1519/JSC.0b013e318 $1 \mathrm{c} 6 \mathrm{a} 128$

Selkow, N. M., Pietrosimone, B. G., and Saliba, S. A. (2011). Subcutaneous thigh fat assessment: a comparison of skinfold calipers and ultrasound imaging. J. Athl. Train. 46, 50-54. doi: 10.4085/1062-6050-46.1.50

Thornton, M. K., and Potteiger, J. A. (2002). Effects of resistance exercise bouts of different intensities but equal work on EPOC. Med. Sci. Sports Exerc. 34, 715-722. doi: 10.1249/00005768-200204000-00024 
Zagatto, A. M., Bertuzzi, R., Miyagi, W. E., Padulo, J., and Papoti, M. (2016). MAOD Determined in a single supramaximal test: a study on the reliability and effects of supramaximal intensities. Int. J. Sports Med. 37, e9. doi: $10.1055 / \mathrm{s}-0036-1582330$

Conflict of Interest: The authors declare that the research was conducted in the absence of any commercial or financial relationships that could be construed as a potential conflict of interest.

Publisher's Note: All claims expressed in this article are solely those of the authors and do not necessarily represent those of their affiliated organizations, or those of the publisher, the editors and the reviewers. Any product that may be evaluated in this article, or claim that may be made by its manufacturer, is not guaranteed or endorsed by the publisher.

Copyright (c) 2021 João, Almeida, Tavares, Kalva-Filho, Carvas Junior, Pontes, Baker, Bocalini and Figueira. This is an open-access article distributed under the terms of the Creative Commons Attribution License (CC BY). The use, distribution or reproduction in other forums is permitted, provided the original author(s) and the copyright owner(s) are credited and that the original publication in this journal is cited, in accordance with accepted academic practice. No use, distribution or reproduction is permitted which does not comply with these terms. 